\section{THE BENEFICIAL IMPACT OF SCHWARZ ROUNDS FOR STAFF: 3 YEARS' EXPERIENCE AT A TERTIARY REFERRAL CANCER CENTRE}

M McNulty, I Droney, AM Stevens, A Halley. The Royal Marsden NHS Foundation Trust

\subsection{6/bmjspcare-2019-ASP.119}

Background Schwartz Rounds (SR) aim to provide a structured forum for staff to discuss the psychosocial and emotional issues of work in healthcare. Patient safety is linked with staff wellbeing, with potential associations between burnout and medical mistakes, and possible impact on effective organisational response to change. These issues are particularly relevant to the NHS and other healthcare systems currently.

Aims This service evaluation sought to explore the relevance and impact of SRs for healthcare staff.

Methods Retrospective analysis of feedback forms from staff who attended SRs from March 2015 to June 2018 at a tertiary referral cancer centre. Descriptive statistics were used to describe staff and their agreement with statements about their experience. Thematic analysis of free text comments was conducted.

Results 1091 feedback forms were analysed from 28 SRs with 3449 attendees (31.6\% response rate). 963 (88\%) agreed with the statement 'Today's Round will help me work better with my colleagues'. The main staff groups represented were: Nursing (36\%), AHP's (29\%) and administration (17\%). Doctors represented 7\%. 71\% respondents disclosed if they had attended a SR before. Of those, $49 \%$ had been to between 1 and 5 SR, while $43.5 \%$ had never attended. $21 \%$ of respondents gave comments which were analysed for qualitative data. $81 \%$ positive, $3 \%$ negative, $4 \%$ neutral and $12 \%$ other comments were made. Keywords used by staff to describe the SR's emerged; insightful, reflection, interesting, inspiring, sharing, supportive. Poignant comments described relief from feelings of isolation and having been given the courage to keep going'.

Conclusion Our evaluation of 3 years' experience demonstrates that SRs are highly acceptable to staff who attend, as a means of interactive and supportive reflection. Qualitative data revealed that most staff described SRs in positive terms. Our evaluation supports evidence that SRs can be a source of support for staff.

\section{COMPLIANCE WITH LOCAL ANTIMICROBIAL GUIDELINES IN AN INPATIENT HOSPICE UNIT}

Sarah Sneller, Bethany Wright. St Peter's Hospice (Bristol)

10.1136/bmjspcare-2019-ASP.120

Background Hospice patients are extremely susceptible to infection and the decision to use antimicrobials is multifactorial. Compliance with the inpatient hospice unit's (IPU) antimicrobial guidelines, adapted from local acute trust and community guidelines, based on Public Health England's (PHE) antimicrobial stewardship principles, was audited.

Method A retrospective review of notes and drug charts was conducted for patients admitted to IPU between February and May 2018, who were given antimicrobials. Data from electronic notes and drug charts was collected and compared to the local hospice antimicrobial standards.
Results Over 4 months, there were 87 admissions to IPU, with 46 systemic antimicrobial prescriptions in 23 patients. Compliance with documenting patient information and recording drug allergies was excellent (100\% and 91\% respectively). Documenting an indication for the chosen antimicrobial in the drug chart and documenting the course duration or review date in the notes had a poor compliance (30\% and 17\%). Other standards included documenting an indication for the chosen antimicrobial in the notes (83\%), documenting the course duration or review date in the drug chart (67\%) and choosing the appropriate antimicrobial based on local guidelines (67\%).

Discussions to improve compliance have included considerations around updating drug charts and further involvement of pharmacists regarding antimicrobial prescriptions.

Conclusions Antimicrobial guidelines aim to encourage consistent prescribing and documentation to produce safe, effective and economical antimicrobial use. There is evidence here to show decisions to start, review, and stop antimicrobials (in line with the PHEs recommended 'Start Smart - then Focus' approach) in most cases was clearly documented and compliant with local guidelines. However, it is also important to recognise the hospice population have different needs, and therefore total reliance on hospital or community guidelines may not always be appropriate. Decisions to start antimicrobials must continue to be an individualised approach.

\section{STEROID PRESCRIBING IN AN IN-PATIENT PALLIATIVE CARE UNIT: A QUALITY IMPROVEMENT PROJECT}

Mairi Finlay, Jane Boyden. NHS Tayside

10.1136/bmjspcare-2019-ASP.121

Background Steroids are frequently prescribed in palliative care for many different indications. However, use of steroids can also lead to side effects which can negatively affect the well-being of patients. Although there are no local or national guidelines on steroid prescribing in palliative care, on reviewing the relevant literature a number of common standards are identified. ${ }^{1}$

- Steroids should be prescribed for a recognised indication;

- a plan should be in place for review of the steroid prescription;

- steroids should be prescribed before $2 \mathrm{pm}$ in order to prevent insomnia;

- GI protection should be prescribed, particularly if concurrent NSAID or history of peptic ulcer disease.

Methods Using these standards records from patients admitted to an inpatient palliative care unit over a 1 month period were examined retrospectively, focusing on documentation at the time of admission (if the patient was already prescribed steroids) or at the time of first steroid prescription.

Results Of 20 patient records examined 12 had been prescribed steroids. 9 of these 12 were already prescribed steroids at the time of admission to CMC. All patients were prescribed steroids prior to $2 \mathrm{pm}$ and all patients had a prescription for GI protection. 5 patients $(42 \%)$ had an indication for steroids recorded and 3 patients $(25 \%)$ had a plan for review of steroid prescription recorded.

Conclusion These results demonstrate there is scope to improve the recording of the indication and plan for review 